\title{
Chronic phase of COVID-19: physical therapist's challenges in the face of neurological events
}

\author{
Fase crônica da COVID-19: desafios do fisioterapeuta diante \\ das manifestações neurológicas
}

The COVID -19 began in the city of Wuhan, China, in December 2019, and it's caused by a newly discovered coronavirus, the SARS-COV-2. Soon after, the COVID-19 epidemic in China became a pandemic with significant burdens on healthcare and the world economy [1]. COVID-19 presents mainly as a respiratory disease, which is sometimes accompanied by gastrointestinal and musculoskeletal events [2]. According to clinical studies in Asia, the most prevalent symptoms consist of fever, cough, dyspnea, sputum production, myalgia, arthralgia, headache, diarrhea, runny nose, and sore throat $[3,4]$.

A retrospective study in China with more than 200 participants found that a subset of patients with COVID-19 had neurological symptoms [5]. These include impaired consciousness, acute cerebrovascular disease and musculoskeletal symptoms, suggesting the impairment of the central (CNS) and peripheral (PNS) nervous systems. The symptoms observed were more likely to be present in patients who suffered from the severe form of the disease. Another study of the same group reported that in 13 patients COVID-19 infection was followed by cerebrovascular disease, including ischemic stroke, cerebral venous sinus thrombosis (CVST), and cerebral hemorrhage [6]. The expansion of the COVID-19 infection in Europe highlighted an atypical presentation of the disease: patients with olfactory and gustatory disorders (hyposmia and hipogeusia, respectively), characterizing the involvement of the PNS.

Although neurological COVID-19-related events are just beginning to be adequately studied, it is highly likely that some of these patients, particularly those suffering severely from the disease, present CNS impairment as well as neurological events such as those mentioned above. Precise and targeted documentation on neurological symptoms, detailed clinical practice, patients' neurological and electrophysiological investigations, attempts to isolate SARS-CoV-2 from cerebrospinal fluid and autopsy of COVID-19 victims can clarify the role played by this virus in neurological dysfunctions. This poses challenges for the rehabilitation of post-COVID patients.

Being placed as the most evident neurological manifestation of COVID-19, and due to its classic risk factors, stroke constituted an increase in the number of patients trying to find physical therapy services, since new stroke events did not stop happening and continue to be the biggest cause of functional disability. These are people whose consequences are impairments in their muscle tone and strength, coordination, gait, and in the performance of their daily activities. Considering that so far the indications are neurological deficits generated by a stroke, but not only that, in more severe cases of COVID the rehabilitation needs greater dedication, frequency, and specialized and individualized physical therapy. 
After hospital discharge, these patients are referred to an outpatient physical therapy service. In times of social isolation, as a preventive measure against COVID-19, there is a real and significant difficulty in accessing such service. At the same time, the patient in question is considered as part of a risk group, having to systematically prevent themselves from being in contact with third parties. Their return to functionality becomes evident. Impairments, such as stroke and polyneuropathies after extended time in the intensive care unity, are unique conditions for a neurofunctional physical therapy support.

Given this situation of need for social isolation recommended by the World Health Organization (WHO), and necessary care by healthcare professionals, such as the use of PPE (personal protective equipment), two therapeutic possibilities are the use of telemedicine for consultation and monitoring, as authorized by the Federal Council of Physical Therapy [7]. These measures help to meet the need imposed by the pandemic, as well as enable the early initiation of physical therapy for these individuals. At the same time, there may be clinical cases of severe neurological deficits, where the physical therapist's face-to-face performance is essential, which would have to be carried out in a home environment with a professional taking all appropriate measures for individual and patient protection.

In this pandemic scenario, neurofunctional physical therapy faces since the initial phase of social distancing another relevant challenge: meeting the needs of patients who were undergoing physical therapy. Progressive or even sudden neurodegenerative health conditions, such as those that cause trauma to the nervous system, stress the need for physical therapy continuity. When treatment is suppressed, losses and/or setbacks on patient outcomes are evident, the walking ability may be lost, spasticity exacerbates, muscle weakness also, and limited functional activities, reflecting negatively on patient's daily life and quality of life.

Ultimately, the conclusion is that a new spectrum of specialized clinical monitoring of these patients calls the attention of neurofunctional physical therapy professionals. The eminent need to promote the return to full functionality of patients cured of COVID-19, as well as continuity, progress and control of patients already in rehabilitation, is imposed as an increasing demand. It's now up to physical therapists to get updated and ready for action!

Luciana Protásio de Melo (i)*

* Researcher at the Laboratório de Inovação Tecnológica em Saúde (LAIS), at the Universidade Federal do Rio Grande do Norte (UFRN), and professor at UNIFACEX, Natal, RN, Brazil 


\section{References}

1. Epidemiology Working Group for NCIP Epidemic Response, Chinese Center for Disease Control and Prevention. Zhonghua Liu Xing Bing Xue Za Zhi. 2020;41(2):145-51.

2. Huang C, Wang Y, Li X, Ren L, Zhao J, Hu Y, et al. Clinical features of patients infected with 2019 novel coronavirus in Wuhan, China. Lancet. 2020;395(10223):497-506.

3. Young BE, Ong SWX, Kalimuddin S, Low JG, Tan SY, Loh J, et al. Epidemiologic features and clinical course of patients infected with SARS-CoV-2 in Singapore. JAMA. 2020;323(15):1488-94.

4. Wan S, Xiang Y, Fang W, Zheng Y, Li B, Hu Y, et al. Clinical features and treatment of COVID-19 patients in Northeast Chongqing. J Med Virol. 2020;10.1002/jmv.25783.
5. Mao L, Jin H, Wang M, Hu Y, Chen S, He Q et al. Neurologic manifestations of hospitalized patients with coronavirus disease 2019 in Wuhan, China. JAMA Neurol. 2020;e201127.

6. Li Y, Wang M, Zhou Y, Chang J, Xian Y, Mao L, et al. Acute cerebrovascular disease following COVID-19: a single center, retrospective, observational study. SSRN Journal. 2020. doi: 10.2139/ssrn.3550025

7. Conselho Federal de Fisioterapia e Terapia Ocupacional (COFFITO). Resolução no 516, de 20 de março de 2020 Teleconsulta, Telemonitoramento e Teleconsultoria [cited 2020 May 22]. Available from: https://www.coffito.gov. $\mathrm{br} / \mathrm{nsite} / \mathrm{p}=15825$ 